\title{
Coherence in Quantum Transmission Protocols
}

\author{
Pedro H. P. de C. Alvarez*, Marcos C. de Oliveira.
}

\section{Abstract}

We analyse how coherence evolves in quantum transmission protocols, such as quantum teleportation and superdense coding, while the message is being transmitted. Then we derive a basic equation that gives the minimum amount of infrastructure needed to create a quantum transmission protocol, with this tool we generalize teleportation and superdense coding for any amount of information and analyze how the coherence behaves when we send large amounts of information.

\section{Key words:}

Quantum Circuits, Quantum Coherence, Quantum Information.

\section{Introduction}

The two most known methods to exchange information using quantum protocols are quantum teleportation, which uses bits and entangled qubits, which we will define as ebits, to send qubits; and superdense coding, which uses ebits and qubits to send classical bits of information. We want to analyse how these protocols behave when sending great amounts of information, and to do this we looked at how the coherence of these systems evolve throughout the process and how it chances with the amount of information being sent. Then we analyse how special states of message behave in the protocol.

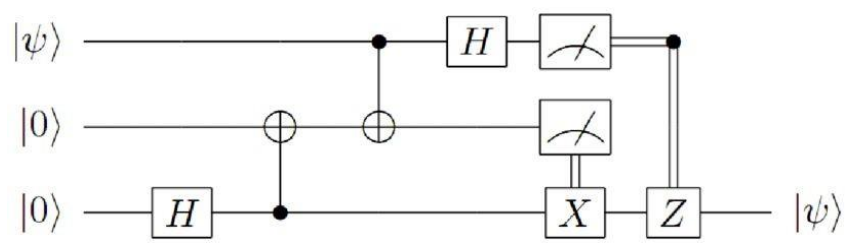

Image 1.Quantum Teleportation Circuit [1].

\section{Results and Discussion}

By analysing the infrastructure needed to setup each of the two types of quantum transmission protocol. We were able to find simple equations that give the basis to construct a quantum transmission protocol, giving the minimum amount of bits, qubits and ebits to send the desired amount of information using an ideal system. Using these equations we created scalable versions of the superdense coding and quantum teleportation protocols. With the scalable superdense coding protocol we discovered that the coherence increases logarithmically with the size of the message. In quantum teleportation the coherence changes with the message following a sine function. Throughout a successful teleportation process the coherence increases and then depletes in the end of the circuit as expected[2]. When scaled, the quantum teleportation protocol showed a linear growth of the coherence with the amount of information sent. Using our results as a basis we were able to develop a simple codification system that allows us to send colors in a qubit. This way we can send pixels and images through quantum teleportation protocols.

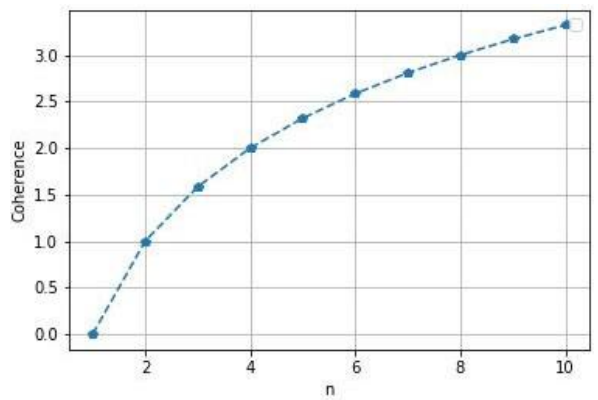

Image 2. Growth of Coherence in Superdense Coding

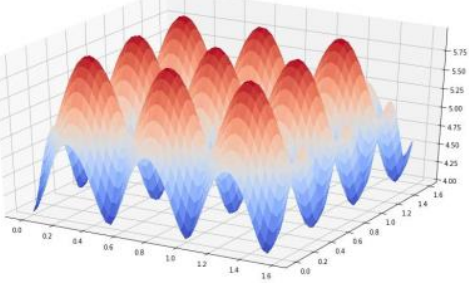

Image 3. Changing of Coherence with teleported message.

\section{Conclusions}

In conclusion, our results show that coherence in simple quantum transmission systems follows simple equations, a logarithm for superdense coding and a linear equation for quantum teleportation, allowing us to understand better the behavior of these systems, and use this information to help in the development of new protocols and technologies, such as the color coding protocol that were mentioned.

[1] Nielsen, M.A.; Chuang, I.L.Quantum Computation and Quantum Information, 10th ed.; Cambridge UniversityPress: New York, NY, USA, 2011.

[2] Liu, Y.; Shang, J.; Zhang, X. Coherence Depletion in Quantum Algorithms. Entropy 2019, 21, 260. 